\title{
L'innocence du romancier : Orhan Pamuk ouvre son art
}

The innocence of the novelist: Orhan Pamuk opens his art

\section{Yan Hamel}

\section{(2) OpenEdition}

\section{Journals}

Édition électronique

URL : http://journals.openedition.org/tangence/602

ISSN : 1710-0305

\section{Éditeur :}

Université du Québec à Rimouski, Université du Québec à Trois-Rivières

Édition imprimée

Date de publication : 1 décembre 2018

Pagination : 61-77

ISSN : 1189-4563

Référence électronique

Yan Hamel, «L'innocence du romancier : Orhan Pamuk ouvre son art », Tangence [En ligne], 118 | 2018, mis en ligne le 01 décembre 2019, consulté le 02 décembre 2019. URL : http://

journals.openedition.org/tangence/602

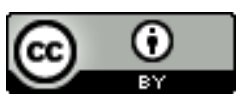

La revue Tangence est mise à disposition selon les termes de la Licence Creative Commons Attribution 4.0 International. 


\section{L'innocence du romancier: Orhan Pamuk ouvre son art \\ Yan Hamel \\ Université TÉLUQ}

C'est avec des romans qu'on fait d'autres romans. La profession de foi en une intertextualité autotélique devrait, dans sa simplicité, suffire à cerner l'art raffiné d'Orhan Pamuk. Voilà du moins ce que laissent croire plusieurs extraits du livre Le romancier naïf et le romancier sentimental, qui transpose à l'écrit le cycle des conférences Charles Eliot Norton prononcées à l'Université Harvard en $2009^{1}$, au cours desquelles le prix Nobel de littérature a voulu «expliquer la façon dont [il] conçoi[t] le genre qu'on appelle "roman". ${ }^{2}$ " Parvenu au faite de sa reconnaissance internationale au double titre de créateur et de théoricien de sa propre pratique artistique, l'auteur fonde l'évolution de sa carrière sur une sorte de fantasme originaire: un Stambouliote de 22 ans qui avait jusque-là espéré devenir peintre

1 . Ces conférences ont été prononcées à l'automne 2009. L'ouvrage qui en a été tiré est originalement paru en anglais, sous le titre The Naive and Sentimental Novelist (Harvard, Harvard University Press, 2010). Fondées en 1925, les Charles Eliot Norton Lectures sont un cycle annuel de six conférences données à l'Université Harvard. Ce sont en majorité des écrivains, mais aussi à l'occasion d'autres artistes, qui ont été conviés à partager leur vision de l'art et de l'esthétique. On a pu notamment y entendre T. S. Eliot, Igor Stravinski, Erwin Panofsky, E. E. Cummings, Jorge Luis Borges, Octavio Paz, Northrop Frye, Italo Calvino, John Cage, Umberto Eco et George Steiner.

2. Orhan Pamuk, Le romancier naïf et le romancier sentimental, trad. de l'anglais par Stéphanie Levet, Paris, Gallimard, coll. «Arcades», 2012, p. 71. Désormais, les références à cet ouvrage seront indiquées par le sigle $R N$, suivi de la page, et placées entre parenthèses dans le corps du texte. 
décida subitement de troquer les pinceaux pour la plume; devant faire face à son entourage, qui lui reprochait de vouloir écrire sans rien connaître de l'existence, il se persuada qu'on "n'écrit pas des romans parce qu'on pense comprendre la vie et les gens, mais parce qu'on pense comprendre les autres romans et l'art du roman, et qu'on désire écrire de la même façon» $(R N, \mathrm{p} .157)$. Trente-cinq ans plus tard, loin de désavouer le novice qu'il fut, le conférencier de Cambridge se rattache avec davantage d'assurance encore à la catégorie des «écrivains qui sont fascinés par l'artificialité du texte et son impuissance à atteindre la réalité, et qui prêtent une attention scrupuleuse aux méthodes mises en œuvre dans l'écriture des romans et aux processus mentaux mis en jeu dans la lecture» ( $R N$, p. 20). Franchissant un pas de plus, il ajoute que, si les romans déjà écrits sont les outils grâce auxquels chacun peut créer ses propres œuvres, ils sont aussi, aux côtés des romans à faire, les instruments les mieux conçus pour réaliser le projet intellectuel de saisir ce qui caractérise l'art romanesque: "La meilleure façon d'étudier le roman, c'est de lire les grandes œuvres et d'aspirer à écrire quelque chose qui leur ressemble» ( $R N$, p. 156). En d'autres termes, c'est avec des textes romanesques qu'on fait d'autres textes romanesques, et c'est avec des textes romanesques - les siens et ceux des autres — qu'on réfléchit (à) l'art du roman: il faut devenir soi-même un romancier - un grand romancier réflexif — ou du moins aspirer à le devenir, avant de pouvoir en arriver à réaliser la meilleure des études sur le genre.

On ne se surprendra pas dès lors de voir Pamuk insister dans ses exposés sur la boulimie de lecture à laquelle il est en proie depuis près de cinquante ans, et convoquer, au fil de ses démonstrations, des exemples tirés des œuvres les plus diverses, allant du Dit du Genji à Maîtres anciens, en passant par Don Quichotte, Robinson Crusoé, Tom Jones, Tristram Shandy, La nouvelle Héloïse, Les fiancés, Le père Goriot, Moby Dick, David Copperfield, Les misérables, L'éducation sentimentale, Ulysse, Le fils de la servante, Le maître et Marguerite, Les choses, Si par une nuit d'hiver un voyageur, L'insoutenable légèreté de l'être, et ce, tout en manifestant une vive prédilection pour quelques œuvres-phares signées Stendhal, Dostoïevski, Tolstoï, Mann, Woolf, Proust, Faulkner et Nabokov. On ne se surprendra pas davantage en voyant l'auteur revenir à répétition sur ses propres œuvres, présentées comme autant d'analyses savantes consacrées à quelques-unes des traditions qui ont donné ses principales formes 
au genre romanesque. Les lois propres au grand roman social issu $\mathrm{du} \mathrm{XIX}^{\mathrm{e}}$ siècle français et britannique, ainsi qu'aux fresques familiales à la Buddenbrook, sont examinées par Cevdet Bey et ses fils. Celles du roman autobiographique le sont par Le livre noir. Celles du roman historique par Le château blanc et par Mon nom est rouge (lequel explore aussi les voies du roman choral). Neige travaille sur l'héritage du roman métaphysique et politique héritier des Démons. Et les méandres labyrinthiques du roman expérimental tracés par Robbe-Grillet, Butor ou Nabokov sont redessinés dans La maison du silence $^{3}$. Refusant de se camper dans la position du maître parvenu à fixer son art en une forme achevée et reproductible, comme le font les peintres ottomans du $\mathrm{XVI}^{\mathrm{e}}$ siècle mis en scène dans Mon nom est rouge, seul le romancier en quête d'originalité, curieux de territoires neufs à explorer, est à même de saisir l'art du roman:

Il n'y a pas de formule, de règle établie. Mais je me débrouille pour ne pas écrire deux romans sur le même mode. J'essaie de tout changer. C'est pourquoi tant de mes lecteurs me disent: «J'aime tel de vos romans, c'est une honte que vous n'ayez pas poursuivi dans cette veine», ou bien: "je n'aimais aucun de vos romans jusqu'à ce que vous écriviez celui-là» — je l'ai particulièrement entendu à propos du Livre noir. En fait, je déteste cela. Il est vital d'expérimenter d'autres formes, d'autres styles, de s'inventer un autre langage, une autre persona et de concevoir chaque livre différemment ${ }^{4}$.

Si l'on ne doit pas être surpris de voir Pamuk penser l'art du roman en revenant sur les œuvres romanesques qu'il a lues et sur les recherches formelles qu'il a réalisées en tant que romancier, on pourrait par contre s'étonner de le voir consacrer du temps à présenter des exposés, comme il l'a fait à Harvard, ou à écrire les nombreux essais sur l'art du roman qui avaient auparavant été réunis dans le recueil D'autres couleurs. À quoi peut servir de chercher à saisir l'essence du roman par le biais d'une écriture théorique, critique ou essayistique excédant les limites de la fiction si le genre romanesque suffit à s'engendrer et à se penser lui-même? Tout aussi incongrue apparaît

3. À ce sujet, voir $R N$, p. 158 .

4. Orhan Pamuk, D'autres couleurs, trad. du turc par Valérie Gay-Aksoy, Paris, Gallimard, coll. «Folio», 2011, p. 591. Désormais, les références à cet ouvrage seront indiquées par le sigle $D A C$, suivi de la page, et placées entre parenthèses dans le corps du texte. 
la tendance lourde, chez l'essayiste, à convoquer, pour échafauder ses raisonnements, les noms de certains des théoriciens les plus marquants du xx siècle: Bakhtine, Benjamin, Bourdieu, Chklovski, Eco, Foucault, Habermas, Iser, Lukács, Ortega y Gasset... Idem en ce qui a trait à ses constants retours sur quelques essais signés par les grands écrivains qui ont réfléchi à leur pratique propre: une partie importante de ce que Pamuk avance à propos de la littérature en général et du genre romanesque en particulier récupère des idées proposées par Coleridge, Eliot, Forster, Horace, Lessing, Naipaul, Poe, Sartre, Schiller et Yourcenar. Loin d'être le textualiste archi-radical qu'il laissait pressentir, le romancier ne dédaigne pas, bien au contraire, de s'alimenter aux proses d'accompagnement circonstancielles (préfaces, entretiens...) par lesquelles les auteurs cherchent à élucider le sens de leurs œuvres majeures et à en orienter la lecture:

Comme beaucoup de lecteurs, j'aime lire cette «métalittérature», qui prend parfois une forme théorique, métaphysique ou poétique. Les affirmations et les justifications avancées par les romanciers pour légitimer leurs textes, la singularité de leur langue, leur duplicité, leurs feintes et leurs incohérences, leurs emprunts et leurs sources sont parfois aussi révélateurs que leurs romans euxmêmes. (RN, p. 38)

Ce qui est vrai de ces auteurs-là est aussi vrai de Pamuk, bien sûr. La volonté de fixer une théorie du roman qui anime les différents essais portant sur le genre est perturbée, et dynamisée, par des duplicités, des feintes et des incohérences, qui sont révélatrices, non seulement des oscillations psychologiques et esthétiques propres à l'écrivain turc, mais aussi de tensions qui travaillent en profondeur l'art du roman et la connaissance que nous pouvons en avoir.

Pamuk n'aborde jamais de front la question d'une potentielle division des tâches entre textes romanesques et textes non romanesques. Sa position sur la pertinence de la théorie littéraire et des essais - y compris les siens - pour qui veut penser l'art romanesque se révèle ambivalente, fluctuante; elle ouvre par ses oscillations une première déchirure dans le tissu d'autoréflexivités intertextuelles au sein duquel l'auteur aurait voulu enserrer le roman. L'essentiel de son malaise se donne à lire dans cette boutade: «Bien que j'aie beaucoup appris de la théorie et qu'il m'arrive parfois d'y céder comme à une néfaste addiction, la plupart du temps je pense qu'il vaut mieux 
l'éviter» (DAC, p. 459). D'où viennent cette culpabilité et ce déni? Il est tout de même rare de voir un homme de culture tenir une chose très instructive pour une néfaste addiction, au point de laisser entendre qu'il bataille infructueusement contre lui-même pour parvenir à s'en défaire. Tout se passe comme si l'orgueil du romancier se voulant souverain était miné par les apprentissages sur lui-même auxquels pouvaient seules le porter d'attirantes échappées du côté de la théorie ou de l'essai. Se dévoile une fragilité, une sourde instabilité dans ce désir irrépressible de s'ouvrir au chant des sirènes critiques tout en se gardant à toute force de se perdre en elles. Ailleurs, Pamuk dit autrement le même embarras, en prenant cette fois à revers cette instabilité qui le fait balancer entre la maîtrise d'un genre donné pour totalisant et la passion pour les approches théorique ou critique qui ambitionnent d'analyser ce même genre de l'extérieur. Le vif intérêt suscité par les ouvrages d'études théoriques ou critiques, qui n'apparaît plus comme un motif de honte, est au contraire présenté comme une marque de distinction: «Par rapport à d'autres romanciers que je connais, il me semble que je m'intéresse davantage à la théorie littéraire et que j'aime lire des ouvrages critiques - intérêt qui m'a d'ailleurs été fort utile quand, à l'âge de cinquante ans, j'ai commencé à enseigner à l'Université Columbia» ( $R N, \mathrm{p} .157)$. Le roman selon Pamuk est donc un genre autosuffisant qui ne se suffit pas. Mieux que tout autre genre, le roman permet de comprendre le roman, mais une connaissance approfondie de la théorie et de la critique avantage celui qui entreprend d'enseigner la poétique du genre à l'université. Pour pouvoir établir que le roman se connaît luimême, il faut, paradoxalement, lire et écrire des traités, ou des essais.

Une deuxième ambivalence touche particulièrement, mais sans doute pas exclusivement, ceux qui, comme Pamuk, sont amenés par leur position à examiner le développement du genre à une échelle intercivilisationnelle. Pour l'auteur issu d'Orient, le roman est à la fois mondialement hégémonique et consubstantiellement lié à l'essor de la civilisation occidentale. Cette "grandiose invention» (DAC, p. 371) s'est imposée sur la longue durée comme l'un des principaux foyers d'autoréflexivité grâce auxquels l'Occident a pu à la fois se connaître et se façonner:

Le roman, comme la musique orchestrale et la peinture après la Renaissance, est selon moi l'une des pierres angulaires de la 
civilisation européenne; c'est l'art qui a contribué à décrire et rendre manifeste l'identité de l'Europe, et à faire de cette entité ce qu'elle est. Je parle ici du roman comme mode de réflexion, de compréhension et d'imagination. (DAC, p. 380)

Pour les auteurs qui «vivent en marge de l'Europe et sont aux prises avec la pensée européenne» (DAC, p. 222), le roman conduit à l'universalité, mais dans la mesure où il particularise, voire marginalise doublement. C'est que le roman, d'une part, est une lentille critique européenne: il infléchit la vision que l'écrivain issu d'une autre civilisation peut adopter sur sa société, ses origines, sa culture. À partir du moment où il pense et imagine en romancier, l'écrivain adopte forcément un point de vue qui le décale par rapport au point de vue communément adopté par la majorité de ses contemporains, qui ne lisent et n'écrivent pas d'œuvres romanesques:

Le roman moderne, distinct de l'épopée, est par essence non oriental. Parce que le romancier [turc] est quelqu'un qui se tient à l'écart de la communauté, qui n'en partage pas les instincts de base, qui pense et juge avec une culture différente de celle dans laquelle il vit, il a une conscience différente de celle de la communauté à laquelle il appartient. C'est un outsider, un solitaire. Et la richesse de ses textes provient de sa vision d'observateur extérieur, de voyeur. Dès lors que vous prenez l'habitude de regarder le monde de cette façon et d'écrire à son sujet sur ce mode-là, vous en venez à désirer vous dissocier de la communauté. (DAC, p. 600)

D'autre part, un romancier comme Pamuk est un artisan non européen. Il doit trouver le moyen de mettre à sa main un outil conçu et développé à l'étranger pour répondre à des besoins qui ne pouvaient être les siens. La tradition culturelle et esthétique à laquelle l'écrivain stambouliote se rattache lui interdit de pratiquer naïvement un art qui ne lui appartient et qui ne lui convient pas d'emblée. Le plaçant dans l'inconfort, elle l'incite à chercher les voies modernistes d'une possible ouverture de la forme à de nouveaux horizons, de façon à ce qu'elle puisse incorporer ce qui lui était jusqu'alors incompatible:

En 1985, je suis parti avec mon épouse pour les États-Unis, où j'ai pu découvrir la prééminence et l'immense richesse de la culture américaine. En tant que Turc arrivant du Moyen-Orient et tentant d'acquérir le titre d'écrivain, je me suis senti intimidé. Je me suis alors retourné vers mes racines. J’ai réalisé que ma génération avait 
à inventer une littérature nationale moderne. Borges et Calvino m’ont libéré. La tradition littéraire islamique était marquée par une telle connotation réactionnaire, politique et par l'usage si absurde et démodé qu'en faisaient les conservateurs, que jamais je n'aurais imaginé pouvoir tirer quoi que ce soit de ce matériau. Mais une fois aux États-Unis, j'ai réalisé que je pourrais y revenir dans l'esprit de Borges ou Calvino. (DAC, p. 593)

Se dire en marge pour rejoindre les incontournables que sont Borges et Calvino; se faire moderniste pour mettre en valeur une tradition apparemment hostile à toute modernité; se devoir de maîtriser les bases cardinales d'un savoir théorique approfondi tout en en préservant sa souveraineté de créateur; verser du côté de l'essai pour dire la supériorité du roman: telle est la position pour le moins inconfortable qui force Pamuk dans une spirale perpétuelle de redéfinitions et de réinventions. Une telle polyvalence convient sans doute à un genre plurivoque et multifocal qui sait orchestrer glissements, fluctuations, indécidabilités et dissonances cognitives: "Les romans sont des structures singulières qui nous permettent de n'avoir aucun mal à faire coexister dans notre esprit des pensées contradictoires, et de comprendre simultanément des points de vue divergents» ( $R N$, p. 35). Le roman est un genre en mouvement qui rend identités et catégories poreuses, à commencer par celles qui structurent le psychisme de leurs auteurs:

Le roman est [...] l'art de se raconter comme si nous étions un autre, mais cela n'est qu'un aspect [...]. Quant à l'autre aspect, c'est [...] la possibilité d'écrire sur la vie des autres comme s'il s'agissait de la nôtre. C'est à travers ce type de recherche que les romanciers s'appliquent à transformer la perception des frontières entre nous et les autres. Cet autre devient "nous", et nous devenons un "autre». Un roman fait bien sûr les deux en même temps. Il raconte notre propre vie comme si c'était celle d'un autre et nous offre la possibilité d'écrire la vie d'autres personnes comme s'il s'agissait de la nôtre. (DAC, p. 371)

Que l'«art du roman repose sur notre capacité à croire de façon simultanée à des états contradictoires» $(R N, \mathrm{p} .12)$ explique que la pensée du romancier sur sa propre pratique ne se laisse pas aisément ramener à un sens monologique, si étranger au genre qu'il s'agit de saisir. Le romancier a un esprit romanesque, c'est à dire 
polyphonique. Son écriture essayistique en est rendue ondoyante; elle glisse capricieusement d'une perspective à son contraire, revient sur elle-même pour se prendre à rebours, sans que les imprécisions et les impossibilités logiques n'en perturbent le développement. Le lecteur cherchera en vain dans Le romancier naïf et le romancier sentimental ou dans D'autres couleurs une intellection systématique achevée et close, pour appliquer à un autre contexte les mots fameux de Lukács. Le roman répond aux aspirations d'un type humain particulier, celui qui ne se cantonne pas à une vérité, ce dont Pamuk se montre particulièrement conscient lorsqu'il entreprend d'explorer la dimension engagée du genre:

J'ai toujours beaucoup de mal à exprimer mes opinions politiques de manière claire, vigoureuse et tranchée; j'ai l'impression d'être prétentieux, ou de dire quelque chose qui n'est pas tout à fait vrai. Parce que je sais que je suis incapable de réduire ma conception de la vie à la musique d'une seule voix et à un seul point de vue. Je suis, après tout, un romancier. Un romancier qui s'est donné pour tâche de s'identifier à tous ses personnages, notamment aux mauvais. [...] [J]e sais [...] que maintenir des opinions extrêmes et arrêtées sur la nature des choses et des gens est un exercice difficile. Je pense aussi que de telles pensées contradictoires coexistent simultanément avec les meilleures intentions du monde chez la plupart d'entre nous; le plaisir d'écrire des romans provient en grande partie de l'exploration de cette situation spécifiquement moderne, où les gens se retrouvent toujours en contradiction avec eux-mêmes. (DAC, p. 297)

Le roman politique est un genre limité car la politique implique la volonté résolue de ne pas comprendre ceux qui sont différents de nous, quand l'art du romancier implique la volonté résolue de comprendre ceux qui sont différents de nous. ( $R N$, p. 126)

Il n'en va évidemment pas autrement des opinions esthétiques. Il ne saurait être question d'écrire un essai pour réduire à une opinion «vigoureuse et tranchée» les caractéristiques formelles ou les finalités propres à un genre susceptible d'exacerber, par ses foisonnements, toutes les formes imaginables d'ambiguïtés et d'antinomies.

Et pourtant le contraire est aussi vrai. Affirmer que «l'art du romancier implique la volonté résolue de comprendre ceux qui sont différents de nous », n'est-ce pas une manière d'imposer une opinion arrêtée sur la nature de cette chose, pourtant mouvante, multiple, 
qu'on appelle roman? Quoi qu'il en dise, et c'est bien sûr là une autre de ses grandes ambivalences, Pamuk n'en vient en fait jamais à dépasser pour de bon la volonté de réduire le roman à une essence simple.

Après avoir fait ce constat, je me demande: quel essayiste se penchant sur l'art du roman peut prétendre échapper à cette ambivalence fondamentale? Établir, comme je viens de le faire, que le roman est multiple, ouvert, n'est-ce pas aussi, au bout du compte, une manière comme une autre de le limiter? Sans doute soumis à l'influence prégnante de Bakhtine et de ses disciples, sur lesquels mon esprit a eu tendance à se reposer au cours des vingt dernières années, je me suis facilité à quelques reprises le travail en affirmant tout de go que le roman est un genre plurivoque, multifocal, polyphonique... Il n'est pas facile, et peut-être même pas possible de ne pas laisser, au détour d'une démonstration ou d'une analyse, sa réflexion pousser de force le roman dans une case, même si l'on sait très bien que celle-ci est dans les faits trop petite pour lui.

Quoi qu'il en soit, il apparaît que malgré l'infinitude de ses avatars formels, le roman selon Pamuk reste astreint à une exigence de cohérence compositionnelle: "Il y a des motifs constitués par des personnes, des objets, des histoires, des situations, des croyances, des faits ou événements historiques, et la juxtaposition de tous ces éléments - en d'autres termes, une texture» $(R N, \mathrm{p} .63)$. Cette «texture» ferait graviter l'ensemble des éléments mis en œuvre autour d'un noyau immuable: "Dans les romans bien construits, tout est lié à tout, et c'est ce tissu de relations qui à la fois crée l'atmosphère du livre et indique son centre secret» $(R N, \mathrm{p}$. 29). Peu importe sa forme et sa signification sociale, politique, psychoaffective, morale..., un grand texte romanesque trouverait sa valeur sur les plans cognitif et artistique du fait qu'il serait doté de ce centre — «en d'autres termes, le sujet véritable du roman» $(R N, \mathrm{p} .133)$. Plus précisément - ou plus vaguement encore? - Pamuk invite ses lecteurs à tenir ce centre secret pour « une opinion ou une intuition profonde sur la vie, un noyau de mystère enfoui à l'intérieur, réel ou imaginé» $(R N$, p. 131). Qu'est-ce à dire? Le centre pourrait être réel, mais pourrait aussi être imaginé! Il pourrait être saisi par une lecture critique et rationnelle. Une analyse méthodique de la texture pourrait l'élucider. À moins qu'il ne soit rien de plus qu'un fantasme du lecteur à la recherche d'un sens. Le centre serait alors la projection d'une image 
sur un écran qui, par lui-même, ne signifierait rien. Le lecteur qui ne se penche pas sur les romans de Pamuk à la recherche de leur centre secret, mais sur ses essais, en essayant de saisir la signification exacte de cette notion, doit accepter de demeurer dans l'expectative, avec l'impression que l'essayiste est lui-même en orbite autour d'une obsession, d'un obscur objet du désir appréhendé de loin, d'un seul côté à la fois, et sur lequel il n'a jamais une prise ferme. Considérons, pour nous en convaincre, l'extrait suivant:

Ce qui distingue essentiellement un roman d'un poème épique, d'un roman médiéval ou d'un récit d'aventures traditionnel, c'est cette idée d'un centre. Les romans présentent des personnages bien plus complexes que ceux des épopées; ils s'intéressent à des gens ordinaires et explorent les moindres aspects de la vie de tous les jours. Mais ces caractéristiques et ces pouvoirs, ils les doivent à la présence d'un centre quelque part à l'arrière-plan, et au fait que nous les lisons avec l'espoir de cette présence. ( $R N$, p. 31)

L'existence du centre rendrait possible la présence de personnages à la fois plus complexes et plus ordinaires que les personnages dépeints par les genres narratifs prémodernes ou populaires. Cette distinction fait évidemment sourciller: pourquoi faudrait-il croire que des œuvres sont privées d'un centre pour la seule raison qu'elles racontent les histoires de personnages supposément plus extraordinaires, quoique plus simples, et plus éloignés d'une certaine idée de la réalité quotidienne? Le fait d'être strictement codifié devrait au contraire, du moins dans les œuvres réussies, s'accompagner d'une cohérence plus évidente et, par le fait même, d'un centre fort, plus aisément repérable que celui du roman. Mais peut-être est-ce justement la présence d'un centre fort organisant la composition de l'épopée (ou du roman d'aventures) qui équivaut à une absence de centre, au sens romanesque que Pamuk voudrait donner à ce terme. Car le centre du roman selon Pamuk est tout sauf plein: à la fin de l'extrait cité, l'écrivain vide une nouvelle fois le roman de sa substance en laissant entendre que les pouvoirs et les caractéristiques propres au genre romanesque pourraient dépendre de ceux qui les lisent avec l'espoir d'y découvrir la présence de ce centre. Le roman, en son centre, ce serait le désir du lecteur.

À moins que le centre du genre romanesque ne se constitue dans et par l'élaboration de moyens inédits, propres à attiser toujours, 
chez le lecteur, un désir jamais comblé. Les fluctuations de Pamuk dans ses essais le disent à leur manière détournée - il serait encore plus juste de dire à leur manière spiralée —, sans l'expliciter tout à fait: le roman, essentiellement, est un dispositif savant, retors, d'indications et de contre-indications troubles incitant à la recherche perpétuelle d'un centre qui ne se laisse pas dévoiler parce que, justement, il est présent sans exister. L'écriture du roman trace des volutes autour d'un centre flou, mais extraordinairement attractif, auquel elle attache le lecteur tout en l'en libérant:

En lisant des romans modernes au centre ambigu, en cherchant ce centre, nous comprenons aussi que notre esprit est capable de croire en beaucoup de choses en même temps - et que ni notre esprit ni le monde ne contiennent en fait de centre. La difficulté dans laquelle nous nous trouvons ici vient de ce que nous sommes partagés entre notre besoin d'un centre pour comprendre, et notre désir de résister au pouvoir de ce centre et à sa logique dominante. Nous savons d'expérience que le désir de comprendre le monde comporte une dimension politique; et il en va de même de l'instinct moderne qui offre un équilibre unique entre clarté et ambiguité, contrôle et liberté interprétative, composition et fragmentation peuvent apporter une véritable réponse à ces difficultés insolubles. ( $R N$, p. 150$)$

Écrire un roman, lire un roman seraient deux manières complémentaires de (se) fixer une quête d'insaisissabilité. Ce serait donc bien avec les romans déjà écrits, c'est-à-dire les pièges labyrinthiques qui ont déjà fonctionné, et dont il faut savoir se démarquer, que le romancier pourrait faire de nouveaux romans, et ce seraient ces romans à écrire qui indiqueraient avec le moins d'imprécision où semble en être rendue, dans quelle direction semble pouvoir tendre l'essence du genre, qui est un mécanisme toujours réinventé pour faire croire à la présence d'une absence. De ce point de vue, le romancier réfléchissant en essayiste à l'art du roman chercherait à obtenir l'équivalent d'un arrêt sur image. Il voudrait parvenir à montrer — se montrer à lui-même au premier chef —, quels sont les éléments constitutifs de la poétique du genre qui peuvent encore avoir les apparences de la stabilité, et ce, pour en venir à mieux les attaquer de l'intérieur. L'essai sur l'art du roman ne serait pas tant destiné à connaître l'état du genre qu'à le remettre en branle: les découvertes auxquelles il donne lieu auraient pour fin immédiate de se voir falsifiées, et donc 
dépassées, par l'écriture d'un roman nouveau. Enté sur la théorie qu'il défait, le roman inédit ouvre le genre sur des horizons inconnus qui seront, pour un temps, inappréciables, et indépassables, jusqu'à ce que le romancier se refasse essayiste pour réviser sa réflexion... Voilà je pense pourquoi tant d'auteurs de romans parmi les plus novateurs éprouvent un si continuel besoin d'accompagner leurs créations romanesques d'une réflexion théorique ou essayistique. Voilà aussi pourquoi tant de ces réflexions n'aboutissent pas à des résultats qui pourraient être, du point de vue de la théorie critique universitaire, pleinement concluants. Le romancier qui peine sur l'art du roman ne veut surtout pas en venir à un achèvement.

Tels sont les essais sur les grands romanciers et sur le genre romanesque réunis dans D'autres couleurs: ils ont jalonné l'écriture des romans de Pamuk, allant de Cevdet Bey et ses fils à Neige. Telles sont aussi les conférences Charles Eliot Norton, dont la cinquième porte sur un sujet inusité, le roman et le musée, ce qui n'a en fait rien d'étonnant: Pamuk finalisait au même moment le projet du Musée de l'innocence, à la fois roman et musée, auquel il a, cinq ans plus tard, ajouté un catalogue illustré ${ }^{5}$ et, encore trois ans plus tard, un long-métrage de docufiction ${ }^{6}$. Le romancier naïf et le romancier sentimental fait état d'une compréhension du genre romanesque qui est presque simultanément dépassée par la sortie de l'œuvre nouvelle.

Pamuk, comme bien d'autres avant lui, avait depuis longtemps compris que le roman est un genre à tendance impérialiste, s'accaparant toutes les formes textuelles se trouvant sur son chemin:

Mallarmé disait vrai avec cette phrase: «Tout, au monde, existe pour aboutir à un livre.» Et le roman est sans nul doute le type de livre le mieux à même d'absorber tout ce qui existe dans le monde. Depuis des siècles, ce sont encore les romans qui ont donné à l'imagination — en tant que capacité à comprendre les autres, qui est le plus grand talent de l'humanité — sa plus authentique et sa meilleure expression. (DAC, p. 385)

5. Orhan Pamuk, L'innocence des objets, trad. du turc par Valérie Gay-Aksoy, Paris, Gallimard, 2012. Désormais, les références à cet ouvrage seront indiquées par le sigle $I O$, suivi de la page, et placées entre parenthèses dans le corps du texte.

6. Grant Gee (réalisateur), The Innocence of Memories. Orhan Pamuk \& Istanbul, narration originale écrite par Orhan Pamuk, long métrage, 97 minutes, GrandeBretagne, 2015. 
Et ses œuvres ont fait la preuve qu'elles étaient destinées à exercer ce pouvoir de phagocytose, notamment sur les genres et les traditions scripturales turcs (tandis que Le livre noir s'incorpore les chroniques typiques des journaux stambouliotes de la seconde moitié $\mathrm{du} \mathrm{xx}^{\mathrm{e}}$ siècle, Neige met en scène un poète lyrique dont il intègre les œuvres et Mon nom est rouge récupère des fragments d'épopée ottomane, eux-mêmes importés de Perse). Mais un élément semblait devoir rester indépassable: le roman était un dispositif écrit, exclusivement capable d'absorber d'autres dispositifs écrits - à l'exception, notable, mais strictement limitée et souvent superficielle, des textes romanesques illustrés par des photographies, des gravures, des peintures, etc. Par le fait même, le roman, dans sa linéarité obligée, était, comme l'essentiel de la littérature, un art temporel, forcé de laisser le travail sur l'espace et la simultanéité à d'autres disciplines artistiques ${ }^{7}:$ «[L]a poésie (la littérature) est un art qui se déploie dans le temps, alors que la peinture, la sculpture et les autres arts plastiques se déploient dans l'espace. Le Temps et l'Espace sont des catégories kantiennes fondamentales» ( $R N$, p. 85). Jusqu'alors, le roman n'avait pas eu les moyens d'être un art total à la manière de l'opéra wagnérien, ou du cinéma, qui réunissent textes, musiques, éléments visuels divers et jeu dramatique. Le romancier turc, qui avait d'abord voulu être peintre, et qui a ensuite ressenti «à l'égard des peintres» une puissante «jalousie», a toujours, à la lecture des romans, éprouvé pour cette raison «un sentiment d'insuffisance» (RN, p. 107):

[L]es romans ne placent rien de concret devant nous - pas un seul objet à toucher, pas une odeur, pas un son, pas un goût. Quand nous lisons un bon roman, une part de notre esprit pense que nous sommes plongés dans la réalité - et même à un niveau très profond de cette réalité - et que la vie est exactement cette expérience-là. Mais pendant ce temps, nos sens nous informent que rien de ce que nous pensons ne se produit. C'est cette situation paradoxale qui nous laisse insatisfaits.

Plus le roman que nous lisons est puissant et convaincant, plus le sentiment d'insuffisance que nous éprouvons est douloureux. (RN, p. 108)

7. Il y a certes un espace du roman, mais celui-ci est rendu temporel par la linéarité de la langue qui l'expose. 
C'est sur cette double frontière d'un art exclusivement langagier et exclusivement temporel que Pamuk réfléchit dans sa cinquième conférence de Harvard. Et c'est cette même double frontière qu'il entreprenait d'outrepasser grâce à son œuvre hybride Le musée de l'innocence, dont les parties principales ont été conçues «les deux ensemble dès le départ» $(I O$, p. 11).

Le roman et le musée peuvent sans doute être appréciés séparément, comme Pamuk l'indique dans le catalogue:

[L] e jour où le musée fut terminé, j'ai remarqué qu'il avait sa propre âme et existait indépendamment du roman - comme l'auront constaté toutes les personnes venues à Çukurcuma le visiter. [...] [L]e roman et le musée racontent chacun des histoires. Les objets exposés dans le musée correspondent à ceux que je décris dans le roman. Mais les mots sont une chose, les objets en sont une autre. Les images qu'un mot évoque dans notre esprit sont une chose, le souvenir d'un vieil objet que nous utilisions à une époque en est une autre. Néanmoins l'imagination et la mémoire ont beaucoup d'affinités entre elles, c'est la source de la proximité entre le roman et le musée. Cependant, de même que le roman existe par lui-même et se comprend sans le musée, le musée est un endroit que l'on peut visiter et à l'atmosphère duquel on peut goûter tout à fait indépendamment du roman. Le musée n'est pas une illustration du roman, et le roman n'est pas une explication du musée. (IO, p. 18)

Il est vrai que des milliers de lecteurs n'ayant pas eu la chance de se rentre à Istanbul et de franchir la porte du musée peuvent apprécier l'histoire de Kemal, qui est amené par un amour malheureux à se faire collectionneur compulsif et à fonder un musée (situé à l'endroit exact où se trouve le musée véritable). À l'inverse, il est non moins vrai que le visiteur n'ayant aucune connaissance du roman peut être fasciné par le musée et par l'histoire qu'il raconte à sa façon (c'est ce qui m'est arrivé lors d'un voyage de tourisme à Istanbul que j'ai fait en mai 2015). Cependant, les deux parties ne sont pas autosuffisantes. Se contenter de connaître l'une d'entre elles seulement équivaut à n'avoir pas lu chacun des quatre volets formant le Quatuor d'Alexandrie de Laurence Durrell ou chacun de ceux composant la pentalogie Le poids des secrets d'Aki Shimazaki: un lecteur peut toujours apprécier l'un ou l'autre des volumes pris isolément, mais il n'aura qu'une idée partielle de l'univers fictionnel dans lequel 
l'œuvre complète aurait dû le faire pénétrer. Le musée réel est, dans sa matérialité, un élément qui entre à part entière dans la composition du roman, tout comme le roman est l'un des éléments composant le musée - non seulement parce que différentes éditions publiées dans plusieurs langues sont exposées au dernier étage, et parce que des passages lus à haute voix sont intégrés à l'audioguide, mais encore davantage parce qu'un nombre important de visiteurs admirent la collection avec un exemplaire du texte en main, pour en lire ou en relire des extraits tout au long du parcours, réalisant, dans la réalité, l'une des ambitions cardinales poursuivies par le protagoniste: «Ce serait bien [...] que les curieux puissent venir le livre sous le bras visiter mon musée ${ }^{8}$.» Musée et roman composent, à eux deux, un texte.

L'un des objectifs poursuivis par la création de ce dispositif est, mon lecteur l'aura peut-être anticipé, d'accroitre les pouvoirs du réalisme romanesque. Le roman raconte comment le protagoniste Kemal a eu l'idée de contacter le romancier bien connu Orhan Pamuk, à qui il confie le soin de mettre son histoire en récit. Pamuk donne donc de l'intérieur et de l'extérieur du roman, en tant que personnage secondaire et qu'auteur véritable, un sens linéaire à une collection d'objets qui sont à la fois fictifs et bien réels. La dernière salle du parcours muséal est une chambrette munie d'un lit et d'une chaise, sur laquelle Pamuk est censé avoir pris place des centaines de fois pour recueillir les confidences de Kemal. Pamuk, qu'on peut entendre sur l'audioguide de la visite, parle exclusivement, dans la version anglaise, d' "our museum», comme s'il l'avait véritablement conçu en partenariat avec son personnage. Et il fait de même dans le docufiction, où il prend la parole pour raconter, comme si elle était vraie, sa rencontre avec lui et d'autres personnages secondaires du roman ${ }^{9}$. Les photographies illustrant le catalogue officiel du musée présentent de leur côté la «collection de Kemal Basmaci et d'Orhan Pamuk» (IO, p. 58). Constatant que les objets de la fiction romanesque sont réellement exposés dans le musée, ou que les

8. Orhan Pamuk, Le musée de l'innocence, trad. du turc par Valérie Gay-Aksoy, Paris, Gallimard, coll. «Folio», 2011, p. 788. Désormais, les références à cet ouvrage seront indiquées par le sigle MI, suivi de la page, et placées entre parenthèses dans le corps du texte.

9. L'audioguide du musée et le docufiction font également entendre les voix de Kemal et de quelques autres personnages secondaires du roman, interprétés par des acteurs. 
objets réellement exposés dans le musée proviennent de la fiction romanesque, le visiteur doit, selon les vœux de Pamuk, finir par en déduire que Kemal est «réel, lui aussi» (IO, p. 15).

L'entreprise visant à faire entrer le plus complètement possible les objets réels dans l'univers de la fiction, et inversement, concourt aussi, avec les thématiques exploitées et la mise en scène de la vie stambouliote, à réaliser l'une des ambitions fondamentales de Pamuk, qui est, nous l'avons vu, de plier l'outil romanesque occidental aux particularités de la mentalité, de la culture et de la société turques:

Je voulais collecter et exposer dans un musée les «vrais» objets d'un récit fictionnel et écrire un roman fondé sur ces objets. Je ne savais pas encore vraiment à quoi ressemblerait ce musée ni quelle forme prendrait ce roman. Mais je sentais que le fait de me concentrer sur les objets, de raconter une histoire à partir d'eux, rendrait mes personnages différents de ceux des romans occidentaux, que cela leur octroierait un caractère plus stambouliote et plus tangible. (IO, p. 15)

Plus fondamentalement, il s'agit de conquérir, sur le plan de la philosophie esthétique, de nouvelles terres au texte romanesque. Raconter une même histoire par l'entremise d'un musée et par celle d'un roman est, comme le remarque Kemal, une manière de placer en une succession linéaire et continue la multiplicité des objets exposés dans le musée, cet endroit où «le Temps devient Espace» (MI, p. 782): «[L]e trait qui reliait chaque instant était le Temps selon Aristote, je comprenais que le trait qui relierait tous ces objets serait un récit. Autrement dit, je pourrais confier la rédaction du catalogue de mon musée à un écrivain qui y travaillerait comme s'il s'agissait d'un roman» (MI, p. 785). D'autre part, comme le remarque toujours Kemal, l'entreprise vise à spatialiser le texte romanesque, dont tous les éléments muséifiés deviennent lisibles simultanément:

[L'ensemble de la collection, chaque vitrine, chaque pièce doit être visible depuis chaque point de l'espace d'exposition, disait [Kemal]. Comme tous les objets — et donc mon histoire peuvent être appréhendés simultanément de plusieurs points de vue, le visiteur perdra le sens du Temps. C'est la plus grande consolation qui soit. Dans les musées poétiques fondés sur une impulsion du cœur et organisés selon une réflexion rigoureuse, 
c'est parce que le Temps s'annihile que nous trouvons la consolation et non parce que nous sommes en présence des vieux objets que nous aimons. (MI, p. 795)

Le véritable Musée de l'innocence, qui est construit autour d'un escalier central tournant lui-même autour d'une spirale dessinée au sol, correspond à cette description - et il remplit à la perfection la mission qui lui a été assignée par le personnage.

Il n'est pas fortuit que Pamuk délègue à son protagoniste ces remarques sur la nature profonde des significations à la fois métaphysiques, esthétiques et psychoaffectives d'un texte qui est en partie muséal et en partie romanesque. Au cours des conférences de Harvard, le romancier déclarait: «J'ai toujours voulu écrire un livre qui, tout en traitant de l'art du roman, serait aussi une étude en profondeur de l'humanité tout entière, et en particulier de l'homme moderne» (RN, p. 160). Ce livre existe, mais il n'est pas, malgré tout son intérêt, celui qui transpose à l'écrit les conférences de Harvard. Il s'agit plutôt d'une ouvre romanesque ouverte, archi-spéculaire, et vertigineusement réflexive, qui a en partie pignon sur rue, dans le quartier de Çukurcuma, sur la rive européenne d'une capitale d'Orient: Istanbul. 